\title{
Simulador Virtual para Sistema de Navegação Autônoma de Robôs Móveis Utilizando Conceitos de Prototipagem Rápida de Controle
}

\author{
Virtual Simulator for Autonomous Mobile Robots Navigation System \\ Using Concepts of Control Rapid Prototyping
}

\author{
Leonimer Flávio de Melo'; Aziz Elias Demian Junior²; João Maurício Rosário³
}

\begin{abstract}
Resumo
Este trabalho apresenta a proposta de implementação de um ambiente virtual de simulação para projeto e concepção de sistema de supervisão e controle para robôs móveis, que sejam capazes de operar e de se adaptar a diferentes ambientes e condições. Este sistema virtual tem como finalidade facilitar o desenvolvimento de protótipos de sistemas embarcados, enfatizando a implementação de ferramentas que permitam a simulação das condições cinemáticas, dinâmicas e de controle, com monitoração em tempo real de todos pontos relevantes do sistema. Para isso, é proposta uma arquitetura aberta de controle, integrando as duas principais técnicas de implementação de controle robótico em nível de hardware: sistemas microprocessadores e dispositivos de hardware reconfiguráveis. O sistema simulador implementado é composto de um módulo gerador de trajetória, de um módulo simulador cinemático e dinâmico e de um módulo de análise de resultados e erros. O módulo gerador de trajetória tem a finalidade de, uma vez conhecendo-se o ambiente em que o robô irá atuar, com seus obstáculos e particularidades, gerar uma trajetória cartesiana ótima, respeitando os limites e características do robô móvel. Todos os resultados cinemáticos e dinâmicos colhidos durante a simulação podem ser avaliados e visualizados em formatos de gráficos e tabelas, no módulo de análise de resultados, permitindo que seja feito um aperfeiçoamento no sistema, no sentido de minimizar os erros com a otimização dos ajustes necessários. Para a implementação do controlador no sistema embarcado utiliza-se a prototipagem rápida, que é a tecnologia que permite, em conjunto com o ambiente virtual de simulação, o desenvolvimento de um projeto de um controlador para robôs móveis. A validação e testes foram realizados com modelos de robôs móveis não holonômicos de transmissão diferencial.

Palavras-chave: Sistemas robóticos móveis. Sistemas de controle embarcado. Arquitetura de controle reconfigurável. Sistemas de arquitetura aberta. Prototipagem de controle rápida.
\end{abstract}

\begin{abstract}
This work presents the proposal of virtual environment implementation for project simulation and conception of supervision and control systems for mobile robots, that are capable to operate and adapting in different environments and conditions. This virtual system has as purpose to facilitate the development of embedded architecture systems, emphasizing the implementation of tools that allow the simulation of the kinematic conditions, dynamic and control, with real time monitoring of all important system points. For this, open control architecture is proposal, integrating the two main techniques of robotic control implementation in the hardware level: systems microprocessors and reconfigurable
\end{abstract}

\footnotetext{
${ }^{1}$ Prof. Dr. do Departamento de Engenharia Elétrica da Universidade Estadual de Londrina - DEEL-UEL; leonimer@uel.br. 2 Prof. Dr. do Departamento de Engenharia Elétrica da Universidade Estadual de Londrina - DEEL-UEL; aziz@uel.br.

${ }^{3}$ Prof. Dr. da Faculdade de Engenharia Mecânica da Universidade Estadual de Campinas-FEM-UNICAMP; rosário@fem. unicamp.br.
}

Semina: Ciências Exatas e Tecnológicas, Londrina, v. 34, n. 1, p. 81-96, jan./jul. 2013 
hardware devices. The implemented simulator system is composed of a trajectory generating module, a kinematic and dynamic simulator module and of a analysis module of results and errors. The kinematic and dynamic simulator module makes all simulation of the mobile robot following the pre-determined trajectory of the trajectory generator. All the kinematic and dynamic results shown during the simulation can be evaluated and visualized in graphs and tables formats, in the results analysis module, allowing an improvement in the system, minimizing the errors with the necessary adjustments optimization. For controller implementation in the embedded system, it uses the rapid prototyping, which is the technology that allows, in set with the virtual simulation environment, the development of a controller project for mobile robots. The validation and tests had been accomplishing with nonholonomics mobile robots models with differential transmission.

Key words: Mobile robotic systems. Embedded control systems. Reconfigurable control architecture. Open architecture systems. Rapid control prototyping.

\section{Introdução}

Uma das principais motivações deste trabalho é propiciar um ambiente virtual que facilite o desenvolvimento de protótipos de sistemas embarcados, enfatizando a implementação de ferramentas que permitam a simulação das condições cinemáticas, dinâmicas e de controle, com monitoração em tempo real de todos pontos relevantes do sistema. Desta monta, a proposta de um simulador virtual de sistemas robóticos móveis é apresentada juntamente com técnicas de prototipagem rápida.

O termo prototipagem rápida, na sua versão tradicional da engenharia mecânica, designa um conjunto de tecnologias usadas para se fabricar objetos físicos diretamente a partir de fontes de dados gerados por sistemas de projeto auxiliado por computador (CAD) (SARCINELLI FILHO et al., 2002).

No contexto deste trabalho, a prototipagem rápida é a tecnologia que permite, em conjunto com um ambiente virtual de simulação, o desenvolvimento de um projeto de um controlador para robôs móveis. Depois de testado e validado no simulador, o sistema de controle é programado na memória da placa de controle do robô móvel. Desta maneira, uma economia de tempo e material é conseguida, validando primeiramente todo o modelo virtualmente para depois se operar a implementação física do sistema.

\section{Arquitetura Aberta de Supervisão e Controle Embarcada}

$\mathrm{O}$ ambiente proposto é um conjunto de módulos de hardware e software, implementados com ênfase na utilização de processadores DSPs de última geração a nível de controle de trajetória e de lógica reconfigurável a nível de controle local, integrados para dar suporte ao desenvolvimento de protótipos de sistemas embarcados.

A lógica reconfigurável, utilizada neste contexto, apresenta as seguintes justificativas (MELO; ROSÁRIO, 2006):

- Facilidade e rapidez na execução de projeto. Com a utilização de uma série de ferramentas fornecidas pelo mercado é possível a execução, o teste, a simulação e a depuração de um projeto com bastante eficiência. A divisão do projeto em pequenos blocos funcionais permite a melhor distribuição das tarefas em uma equipe de trabalho. Blocos previamente desenvolvidos podem ser aproveitados em novos projetos, minimizando o tempo de execução dos mesmos.

- Minimização de custo. O desenvolvimento de um novo hardware para atender exigências de um novo projeto ou as novas exigências de um projeto antigo, implica em custos que podem ser evitados pela utilização de lógica reconfigurável.

- Fácil expansão. Novos módulos podem ser 
gradativamente adicionados ao sistema original permitindo que novas funcionalidades sejam implementadas sem a necessidade de realizar um novo projeto de hardware.

- Velocidade de operação. Além da característica de operar em paralelo, algoritmos implementados com lógica reconfigurável são tipicamente mais rápidos que algoritmos implementados com lógica convencional (execução de linhas de código através de um processador).

- Domínio tecnológico. Lógica reconfigurável é apresentada como solução para inúmeros problemas práticos de engenharia. Dentro desta realidade é justificável o esforço para dominar está área de conhecimento, buscando suas virtudes e limitações.

A utilização de processadores DSPs na estrutura do sistema a nível de controle de trajetória tem, dentre outras, as seguintes justificativas:

- Velocidade de processamento. Os processadores DSPs têm velocidade de processamento bem superior aos processadores com outras tecnologias, utilizados com freqüência para análise de sinais em tempo real.

- Rapidez na comunicação interna. São rápidos o suficiente para tratar todos os dados recebidos pelos sensores, através dos dispositivos de hardwares reconfigurável.

- Capacidade de processamento. Têm capacidade de processamento suficiente para executar os algoritmos de navegação, de triangulação com marcadores, de geração de trajetórias, inteligência artificial, etc.

São objetivos do ambiente proposto:

- Flexibilidade. O ambiente deve ser capaz de adaptar-se facilmente à aplicação desejada. Um novo conjunto de requisitos deve ser facilmente atendido sem implicar em novos projetos de hardware.

- Expansibilidade. O ambiente deve ser de fácil expansão, permitindo que novas funcionalidades sejam adicionadas a um sistema pré-existente.

- Transparência. O ambiente deve ser totalmente aberto, permitindo a acesso de novos usuários a todos os aspectos do mesmo. Isto vem de encontro as diferentes necessidades acadêmicas de graduação e pós-graduação a que este ambiente visa prestar suporte.

- Adequação a tarefa. O ambiente deve poder ser configurado para resolver problemas de maior ou menor complexidade. Isto permite a utilização adequada de recursos para resolver um determinado problema, evitando o desperdício de recursos.

- Baixo Custo. São considerados aspectos que visem a minimização do custo no desenvolvimento de um protótipo de sistemas embarcados. São utilizadas ferramentas de projeto de baixo custo ou sem custo associado. A própria utilização de lógica reconfigurável permite a minimização do custo total de um projeto por razões já apresentadas anteriormente.

- Capacidade de comando e monitoração remota. Em muitas aplicações de sistemas embarcados a monitoração e modificação de parâmetros internos aos sistemas pode ser de grande utilidade, permitindo um menor tempo de projeto e depuração de erros ou atendendo a prérequisitos deste projeto.

- Estrutura para a prototipagem rápida. Estar estruturado e ser capaz de se comunicar com os sistemas de simulação, como por exemplo, o MatLab, para execução das técnicas de prototipagem rápida e de HIL (Hardware In-theLoop).

O sistema proposto pode ser visualizado como um sistema multi-níveis, dividido em três níveis de controle hierárquicos, conforme ilustrado na Figura 1. 
Figura 1 - Diagrama de blocos ilustrando os níveis de controle para o sistema robótico móvel.

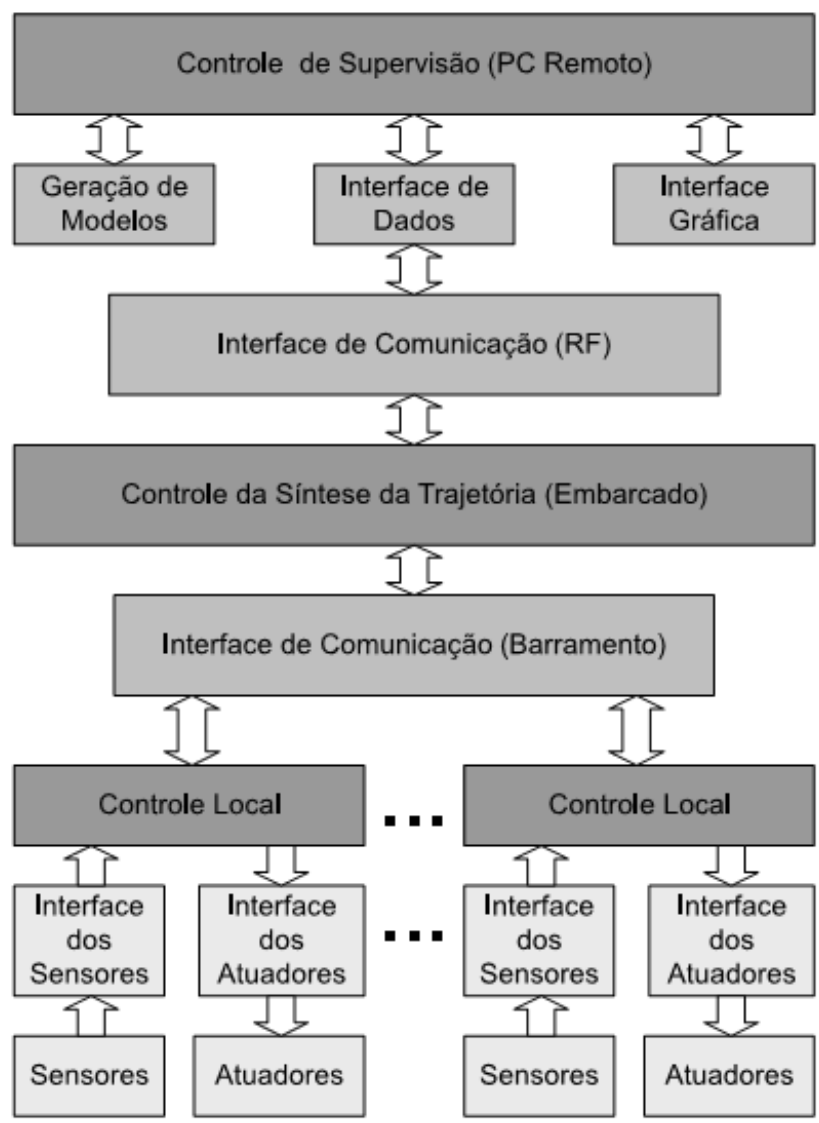

Fonte: (MELO, 2007).

\section{Descrição da estrutura hierárquica}

Nesta aplicação, os blocos de controle locais, as interfaces dos sensores e as interfaces de potência serão implementados através da utilização de PLDs ou FPGAs. A Figura 2 ilustra um descrição dos blocos que serão implementados considerando-se a arquitetura hierárquica e estruturada proposta neste projeto.
Figura 2 - Diferentes níveis de controle do sistema proposto.

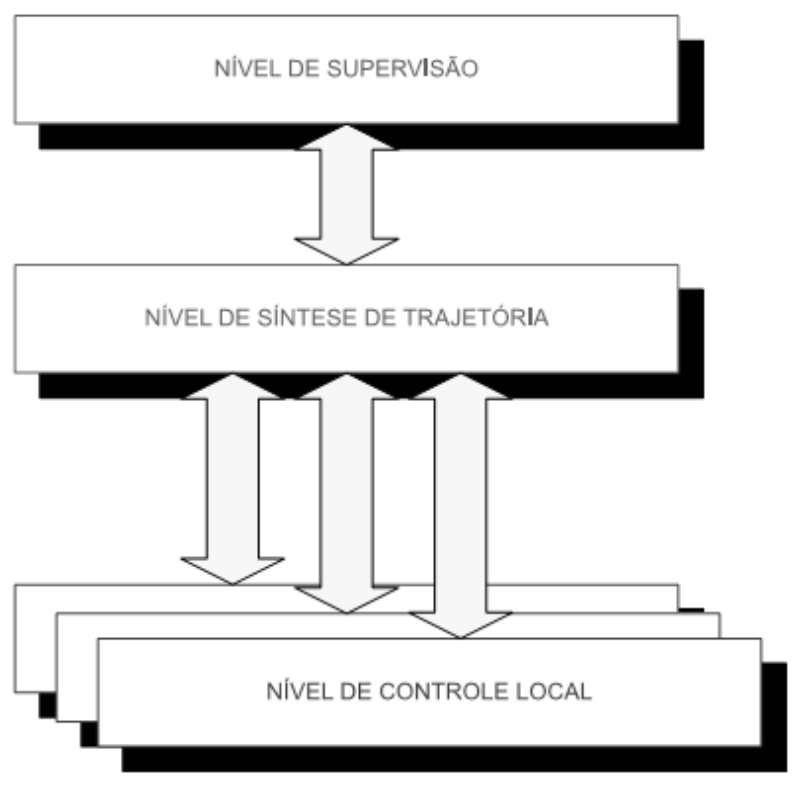

Fonte: (MELO, 2007).

É analisado a seguir, com mais detalhes, a estrutura de cada nível.

- Nível de supervisão. O nível de supervisão deverá ser implementado utilizandose de uma GUI (Graphical User Interface) num sistema Windows com um microcomputador PC. O link de comunicação entre este nível e o nível de síntese da trajetória utilizará a tecnologia de comunicação sem fio por RF (wireless) usada em redes Ethernet com link Wi-Fi. Wi-Fi é um marca licenciada originalmente pela Wi-Fi Alliance para descrever a tecnologia de redes sem fio embarcadas (WLAN) baseadas no padrão IEEE 802.11. Este padrão de comunicação sem fio é utilizado com o protocolo TCP/IP e garante uma taxa de transmissão de até 11 Mbps operando na freqüência de $2,4 \mathrm{GHz}$ e requer poucos acessórios para sua implementação. Hoje em dia, adquire-se placa de interface Wi-Fi a um custo bem acessível haja vista sua popularização na utilização em Lap Tops e microcomputadores. Os 14 canais disponíveis do padrão Wi-Fi em 2,4 
$\mathrm{GHz}$ garantem uma expansibilidade no sistema com a implementação de estratégias de controle de múltiplos robôs.

- $\quad$ Nível de síntese da trajetória. Este nível inclui uma placa de controle da Texas Instruments TMDSDSK6416 DSP board Kit (TEXAS, 2011) que contém o processador DSP TMS320C6416 e todos periféricos necessários para integração do sistema. Esta placa utiliza um sistema de operação em tempo real, que será utilizado para aplicações no sistema embarcado. Através de rotinas em linguagem padrão ANSI C, compatível com o processador, a execução e correção da trajetória associada com outras tarefas definidas pelo nível de supervisão deverão ser realizadas com sucesso.

- Nível de controle local. Como mencionado previamente, este nível inclui todas as interfaces necessárias com os sensores e atuadores, e o controlador de cada motor. Num ponto de vista de uma arquitetura aberta, a implementação destes controladores locais têm que ser gerais o bastante para permitirem uma fácil reconfiguração da lei de controle de acordo com as especificações do usuário.

Para concluir, considere a Figura 3 onde são apresentados detalhes dos blocos que serão implementados em hardware e software, distribuídos de acordo com os níveis de controle propostos (MELO, 2007). O interesse por um projeto modular é óbvio considerando-se um framework para uma arquitetura aberta. Até as leis de controle locais permanecem abertas.
Figura 3 - Representação das estruturas de níveis de controle do robô móvel.

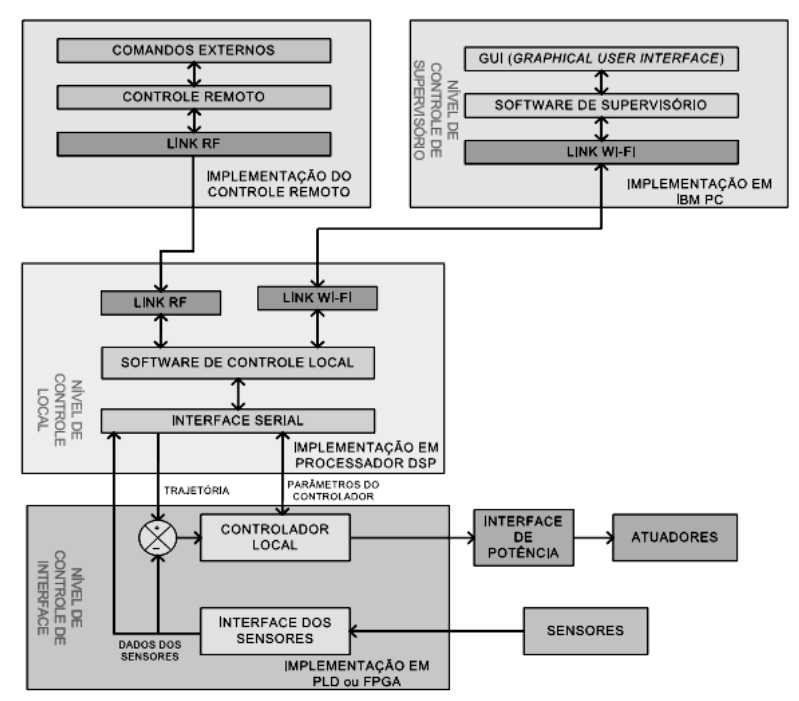

Fonte: (MELO, 2007).

A Figura 4 ilustra a implementação física no robô móvel do sistema proposto, podendo-se observar o lay-out dos dispositivos mecânicos, elétricos e eletrônicos.

Figura 4 - Representação das estruturas de níveis de controle do robô móvel.

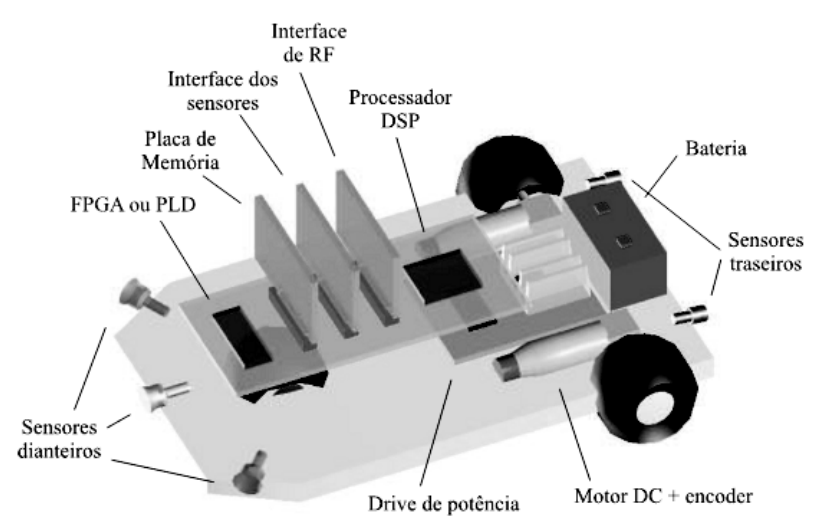

Fonte: (MELO; MANGILI JUNIOR, 2010). 


\section{Modelagem matemática do sistema de controle embarcado}

São apresentas a seguir as implementações dos modelos cinemático e dinâmico utilizados no simulador do robô móvel e na técnica de prototipagem rápida. As equações matemáticas do modelo cinemático adotado têm omo plataforma o sistema robótico móvel não holonômico utilizado como base no desenvolvimento dos modelos cinemáticos e dinâmicos, assim como na implementação do simulador robótico. É apresentado também aspectos relacionados à implementação do controlador PID, utilizado nos controles dos dois eixos, assim como os detalhes de sintonia. A escolha do controlador PID recai principalmente em dois fatores básicos: primeiro, o controle das rodas de tração do sistema robótico é relativamente simples e segundo, a facilidade de implementação em software e hardware deste tipo de controlador. Nada impede que, como estamos tratando de um sistema de arquitetura aberta venham a ser empregados outros tipos de controladores, dependendo da aplicação e da necessidade. Isto porque, todo o sistema foi concebido para ser o mais genérico possível, podendo-se adaptar, com poucas modificações, a qualquer necessidade implementacional.

Para realização de tarefas em nível de sistema de coordenadas (sistema cartesiano) e para geração dos sinais de referência para o controlador de posição de cada junta robótica do sistema mecatrônico em estudo, torna-se necessário o estabelecimento de um modelo matemático baseado na cinemática do sistema. Portanto, o controle de um robô necessita de procedimentos para transformar os dados de referência de posicionamento, tais como a velocidade linear e o raio de curvatura, em coordenadas cartesianas, quando se deseja realizar o controle através de um referencial cartesiano. A Figura 5 ilustra a estrutura de controle do robô móvel com os blocos representativos da geração de trajetória, modelo dinâmico e cinemático do sistema.
Figura 5 - Representação das estruturas de níveis de controle do robô móvel.

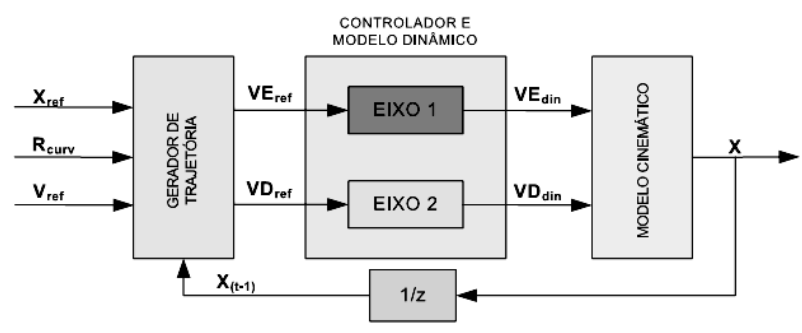

Fonte: (MELO; MANGILI JUNIOR, 2010).

$\mathrm{O}$ gerador de trajetória recebe os dados de referência, tais como o vetor posicionamento $\mathrm{X}_{\text {ref }}=$ $\left[\mathrm{x}_{\text {ref }} ; \mathrm{y}_{\text {ref }} ; \theta_{\text {ref }}\right]$, a velocidade linear de referência do robô $\mathrm{V}_{\text {ref }}$ e o raio de trajetória instantâneo do robô $\mathrm{R}_{\text {curv }}$, que são convertidos em $\mathrm{VE}_{\text {ref }}$ (velocidade linear da roda esquerda) e $\mathrm{VD}_{\text {ref }}$ (velocidade linear da roda direita). Estas velocidades diferenciadas são recebidas pelo controlador, e no modelo dinâmico do sistema, são enviadas às respectivas rodas do robô, através de seus atuadores. São então gerados pelo controlador os vetores $\mathrm{VE}_{\text {din }}$ (velocidade linear dinâmica da roda esquerda) e $\mathrm{VD}_{\text {din }}$ (velocidade linear dinâmica da roda direita). No bloco do modelo cinemático, estes dados são

convertidos no vetor posicionamento final do robô $\mathrm{X}=[\mathrm{x} ; \mathrm{y} ; \theta]$.

\section{Modelo cinemático}

O modelo cinemático implementado no controlador, para movimentos circulares do robô, é descrito na Equação 1:

$$
\left[\begin{array}{l}
x^{\prime} \\
y^{\prime} \\
\theta^{\prime}
\end{array}\right]=\left[\begin{array}{ccc}
\cos (\omega \delta t) & -\operatorname{sen}(\omega \delta t) & 0 \\
\operatorname{sen}(\omega \delta t) & \cos (\omega \delta t) & 0 \\
0 & 0 & 1
\end{array}\right]\left[\begin{array}{c}
x-\mathrm{CCI}_{x} \\
y-\mathrm{CCI}_{y} \\
\theta
\end{array}\right]+\left[\begin{array}{c}
\mathrm{CCI}_{x} \\
\mathrm{CCI}_{y} \\
\omega \delta t
\end{array}\right]
$$

A equação 1 descreve o movimento rotacional do robô a uma distância $\mathrm{R}$ do CCI com um velocidade angular dada por $₫$ (DUDEK; JENKIN, 2000).

Quando o robô está se deslocando em linha reta, 
então $\mathrm{v}_{\mathrm{e}}=\mathrm{v}_{\mathrm{d}}=\mathrm{v}$, com $\mathrm{R} \rightarrow \infty$. O modelo cinemático implementado no controlador é dado obtido a parir da equação 2 (LEONARD; DURRANT-WHYTE, 1992):

$$
\left(\begin{array}{l}
x^{\prime} \\
y^{\prime} \\
\theta^{\prime}
\end{array}\right)=\left(\begin{array}{c}
x+v \cos (\theta) \delta t \\
y+v \operatorname{sen}(\theta) \delta t \\
\theta
\end{array}\right)
$$

Modelagem dinâmica do sistema

O modelo matemático de um sistema dinâmico é definido como um conjunto de equações que representa com precisão ou, pelo menos, razoavelmente bem a dinâmica do sistema. Uma vez obtido o modelo matemático de um sistema, podem ser utilizadas várias ferramentas analíticas e de computação para efeito de análise e síntese. A seguir é apresentada a modelagem dinâmica do atuador utilizado neste trabalho (MELO; MANGILI JUNIOR, 2010).

Cada eixo de tração, localizado nas rodas esquerda e direita do robô móvel, inclui um motor $\mathrm{CC}$, um redutor e um encoder de posição. Considerando as equações de modelagem do motor CC, obtém-se as equações 3 :

$$
\begin{aligned}
e_{a}(t) & =L_{a} \frac{d i(t)}{d t}+R_{a} i(t)+K_{b} \frac{d \theta_{m}(t)}{d t}, \\
T_{m}(t) & =J_{m} \frac{d^{2} \theta_{m}(t)}{d t^{2}}+B_{m} \frac{d \theta_{m}(t)}{d t}, \\
T_{m}(t) & =K_{t} i(t),
\end{aligned}
$$

onde $\mathrm{e}_{\mathrm{a}}(\mathrm{t})$ é a tensão de alimentação da armadura, $\mathrm{T}_{\mathrm{m}}(\mathrm{t})$ o torque do motor, $\theta_{\mathrm{m}}(\mathrm{t})$ a posição angular do rotor do motor, $\mathrm{i}(\mathrm{t})$ a corrente de armadura do motor, $\mathrm{L}_{\mathrm{a}}$ a indutância e $\mathrm{R}_{\mathrm{a}}$ a resistência elétrica da bobina do motor, $\mathrm{B}_{\mathrm{m}}$ é o coeficiente de atrito viscoso, $\mathrm{K}_{\mathrm{b}}$ é a constante de força contra-eletromotriz, $\mathrm{K}_{t}$ a constante de torque e $\mathrm{J}_{\mathrm{m}}$ a inércia do eixo do motor. A Figura 6 mostra o esquema equivalente dos motores $\mathrm{CC}$ utilizados para o tracionamento das rodas do robô móvel.

Figura 6 - Esquema equivalente do motor CC com acionamento da carga.

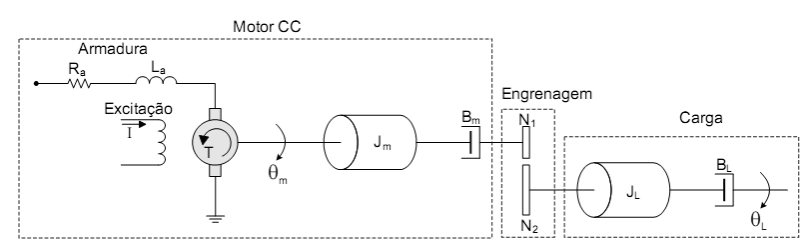

Fonte: (MELO; ROSÁRIO, 2006).

O diagrama de blocos da Figura 7 ilustra o modelamento do motor de CC com o sistema redutor.

Figura 7 - Modelamento do motor CC.

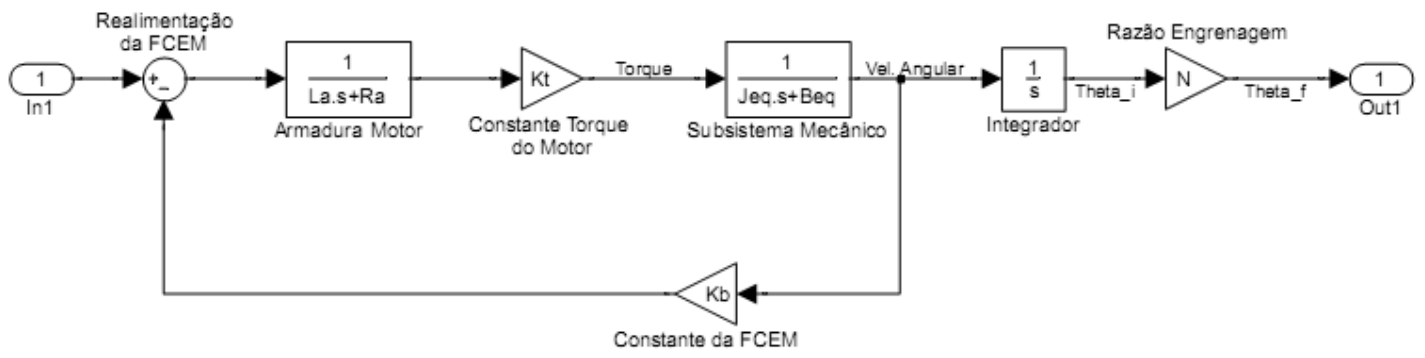

Fonte: (MELO; MANGILI JUNIOR, 2010). 


\section{Controlador PID}

Para controlar a velocidade angular $₫$ dos motores $\mathrm{CC}$ e, conseqüentemente, a velocidade e o deslocamento linear de cada roda, foi implementado um controlador PID. A Figura 8 mostra um diagrama de blocos com a planta e o controlador PID atuando sobre o motor CC.

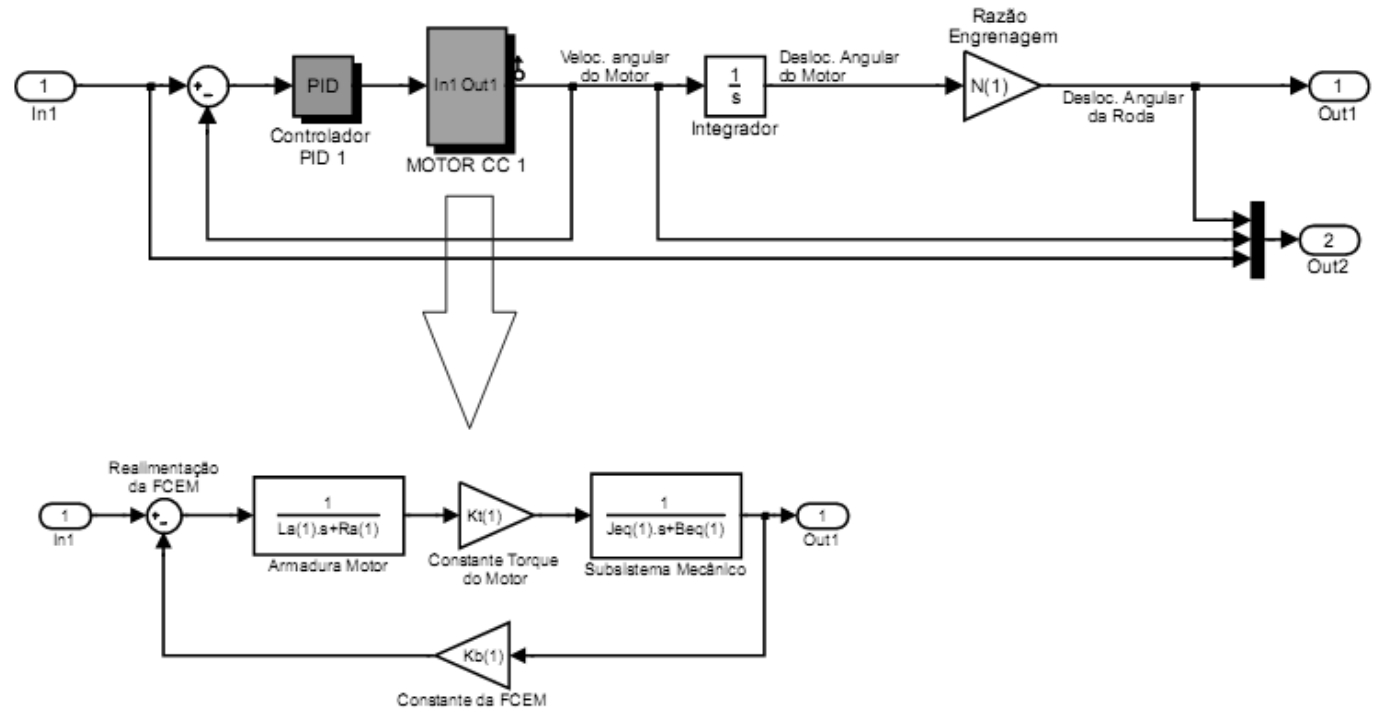

Fonte: (MELO; MANGILI JUNIOR, 2010).

\section{Implementação do simulador virtual}

São apresentadas a seguir as principais características de um simulador de sistemas robóticos móveis. Ele foi implementado a partir do modelo cinemático e dinâmico dos sistemas de acionamento mecânico dos eixos robóticos, para a simulação de diferentes técnicas de controle no campo da robótica móvel, permitindo-se aprofundar os conceitos de sistemas de navegação, planejamento de trajetórias e sistemas de controle embarcado. Esse simulador, implementado em arquitetura modular e aberta, permite a aplicação direta de vários conceitos dentro da área da robótica móvel, sendo utilizado para sua validação, e como objetivo principal deste estudo, o modelo de um protótipo de robô móvel com restrições não holonômicas e tração diferencial com dois graus de liberdade (movimento de deslocamento linear e de rotação).

Para o desenvolvimento do simulador, foram considerados os aspectos construtivos do protótipo do robô móvel, incluindo a modelagem cinemática e dinâmica do sistema de acionamento e controle. O simulador apresenta o módulo gerador de trajetórias que é o primeiro bloco do sistema e foi implementado com a funcionalidade de gerar uma trajetória para o robô móvel a partir de uma matriz de pontos fornecidas inicialmente. Outro bloco apresentado é o controlador implementado na forma PID.

Para a implementação final do controlador utiliza-se o conceito de prototipagem rápida, onde após validação e testes do sistema completo de controle e supervisão através do simulador, a interface de supervisão e hardware de controle poderão ser implementados de maneira rápida, segura e aberta, eliminando custos envolvidos na confecção de protótipos.

A Figura 9 apresenta um visão geral do sistema simulador proposto. A utilização do sistema tem início pela captação de pontos principais para 
geração da trajetória do robô móvel. A idéia é utilizar um sistema de câmara de vídeo ou fotográfico, que capte a imagem do ambiente em que o robô móvel irá navegar. Este sistema inicial deve ser capaz de, através da imagem captada, identificar os obstáculos do ambiente e gerar uma matriz com alguns pontos estratégicos que servirão de entrada para o sistema de geração de trajetória.

Figura 9 - Uma visão geral do sistema implementado.

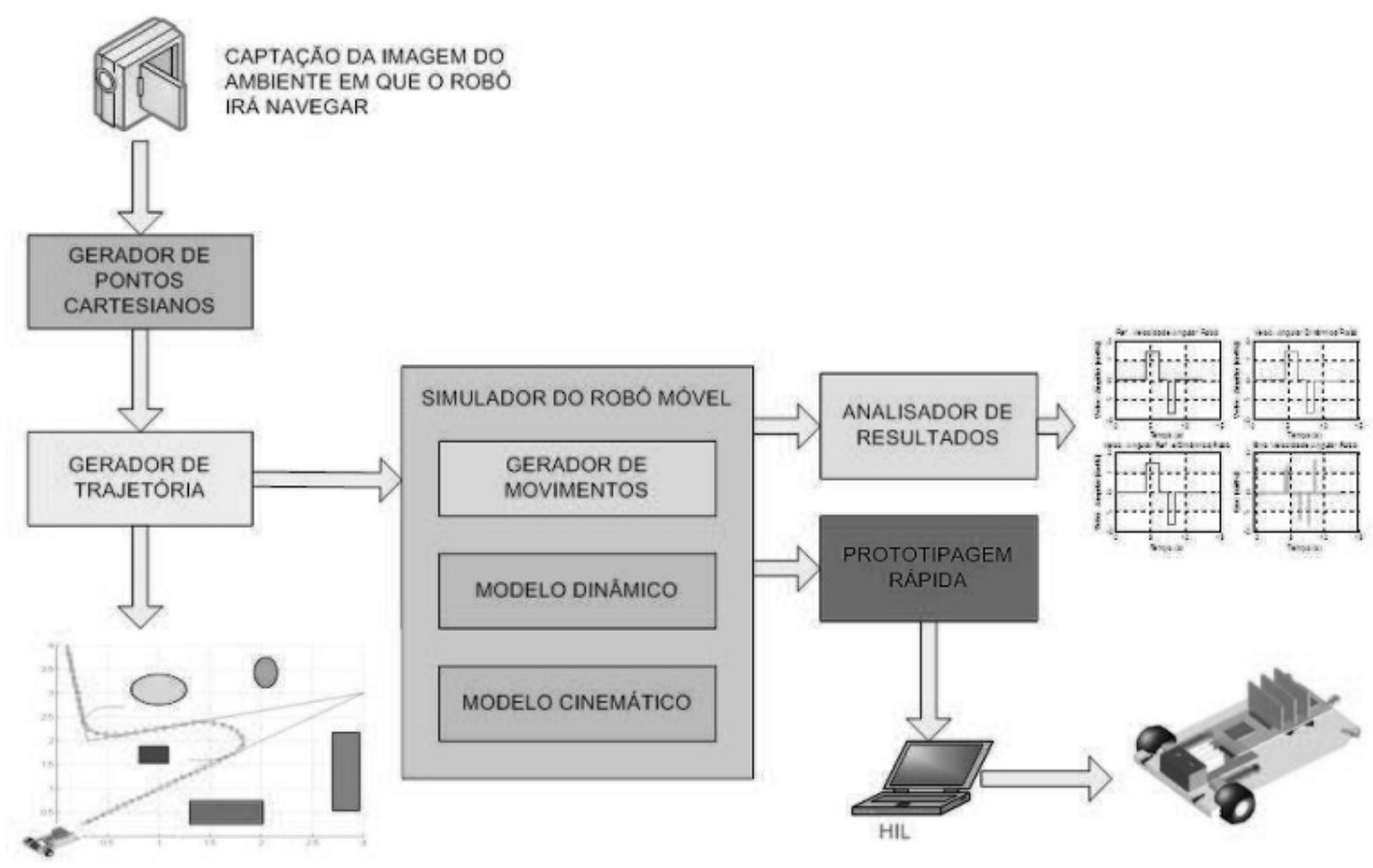

Fonte: (MELO et al., 2010).

A Figura 10 ilustra um exemplo de um ambiente com alguns obstáculos onde o robô deve navegar. Neste ambiente, o robô está localizado inicialmente no ponto P1 e tem como objetivo alcançar o ponto P4. O sistema gerador de pontos cartesianos iniciais, deve então fornecer ao módulo de geração de trajetória, os pontos cartesianos P1, P2, P3 e P4, que são os pontos principais da rota a ser traçada.
Figura 10 - Exemplo de um ambiente com obstáculos para navegação do robô móvel.

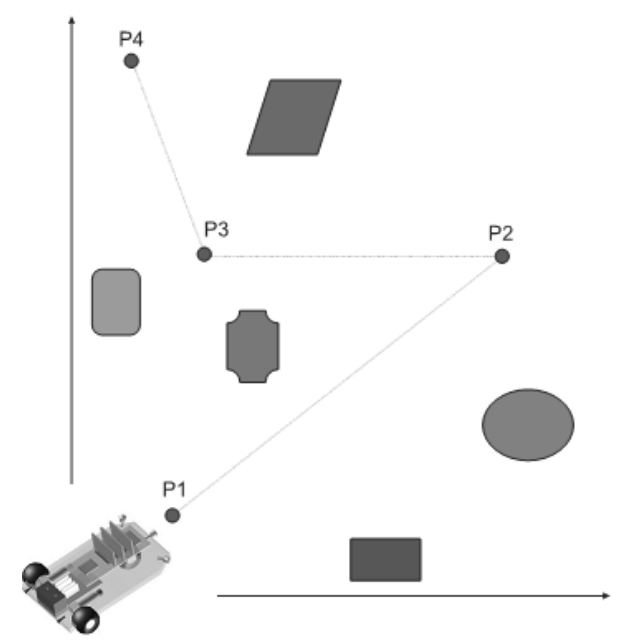

Fonte: (MELO et al., 2010). 
O traçado final da trajetória do robô móvel pode ser observado na Figura 11.

Figura 11 - Traçado final da trajetória do robô móvel.

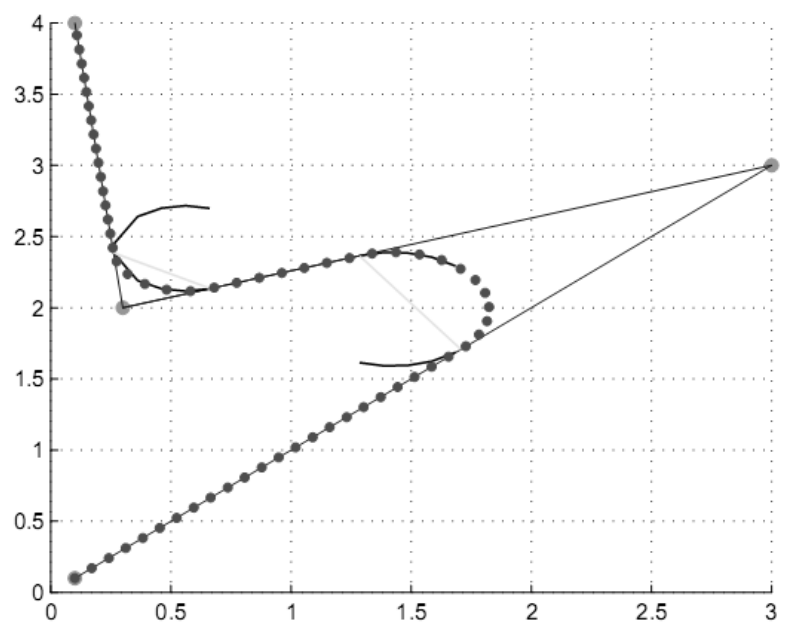

Fonte: (MELO et al., 2010).

\section{Detalhes do simulador virtual}

O sistema simulador virtual do robô móvel é formado por três blocos principais. O primeiro é chamado de bloco de geração de movimentos. $\mathrm{O}$ segundo é o bloco do controlador e modelo dinâmico do robô móvel. O terceiro é o bloco do modelo cinemático. Esta estruturação modular é ilustrada na Figura 12 onde pode-se observar as variáveis de entrada e saída do sistema (MELO; ROSÁRIO, 2006).

Figura 11 - Diagrama de Blocos representando os módulos de geração de movimentos, controlador, modelo cinemático e modelo dinâmico.

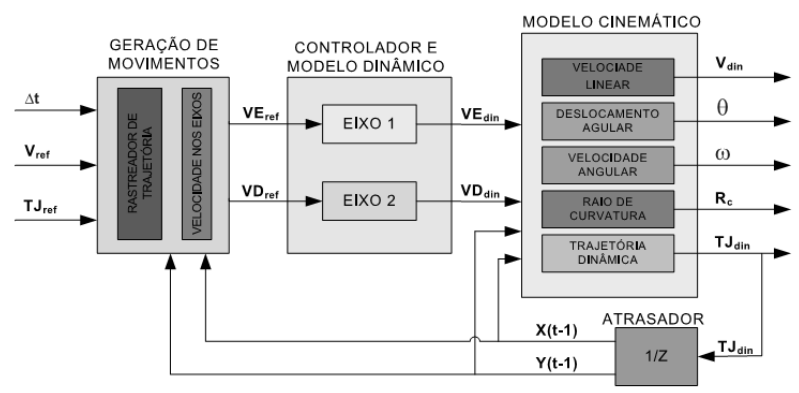

Dentro do bloco do sistema de robô móvel temse o subsistema ilustrado pela Figura 12. Pode-se observar os três principais módulos do simulador do sistema robótico móvel que são o bloco gerador de movimentos, o bloco controlador e modelo dinâmico e o bloco de modelo cinemático.

\section{Resultados das simulações e validação experimental}

São apresentados a seguir alguns dos resultados experimentais, com exemplos de simulações, envolvendo diferentes situações de navegação do um sistema robótico móvel. A validação foi executada em duas etapas distintas, a primeira feita com o simulador virtual obtendo-se analise gráfica de resultados da simulação. A segunda foi feita através de uma implementação experimental do sistema proposto numa plataforma robótica móvel, para testes de cinemática e da dinâmica do sistema. A Figura 13 mostra o robô móvel utilizado nos experimentos.

Figura 13 - Protótipo do robô móvel experimental utilizado nos testes e validações dos resultados.

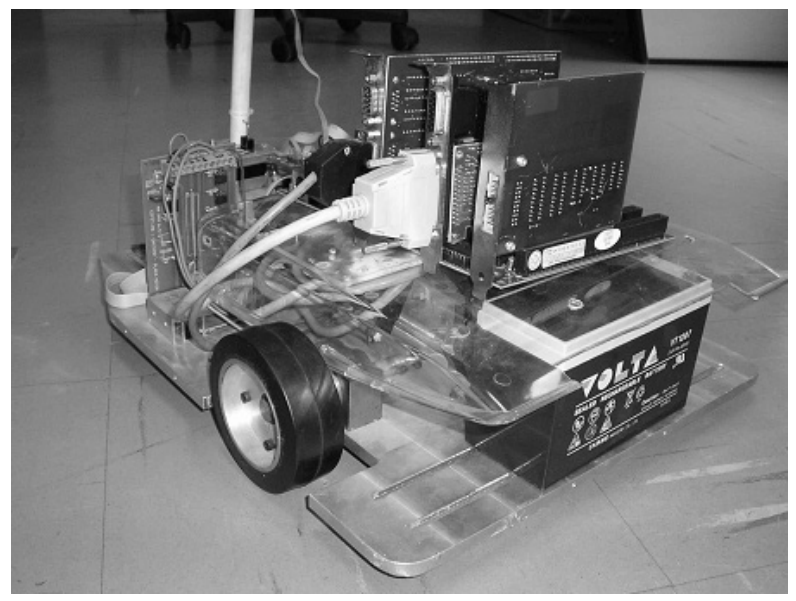

Fonte: (MELO; ROSÁRIO, 2006).

Fonte: (MELO; ROSÁRIO, 2006). 
Figura 12 - Simulador do sistema robótico móvel implementado no Simulink ${ }^{\circledR}$.



Fonte: (MELO; ROSÁRIO, 2006).

O simulador implementado em ambiente Simulink permite a visualização temporal das saídas e entradas do sistema em estudo. Para melhor compreender e analisar o comportamento do sistema torna-se imprescindível a implementação de um analisador gráfico de resultados que é mostrado a seguir. Assim, após as simulações realizadas no domínio do tempo, são obtidos arquivos de dados temporais correspondentes as variáveis de estudo (posição angular e cartesiana, velocidade linear e angular e sinais de controle), que após tratamento conveniente, torna-se possível verificar relevantes resultados para melhor análise do comportamento do sistema.

A seguir, são apresentadas as análises de desempenho e de erros do sistema robótico em questão. A Figura 14 ilustra a trajetória inicial de referência obtida no simulador e a trajetória final dinâmica obtida pelo robô móvel. 
Figura 14 - Trajetória de referência e dinâmica do robô móvel.
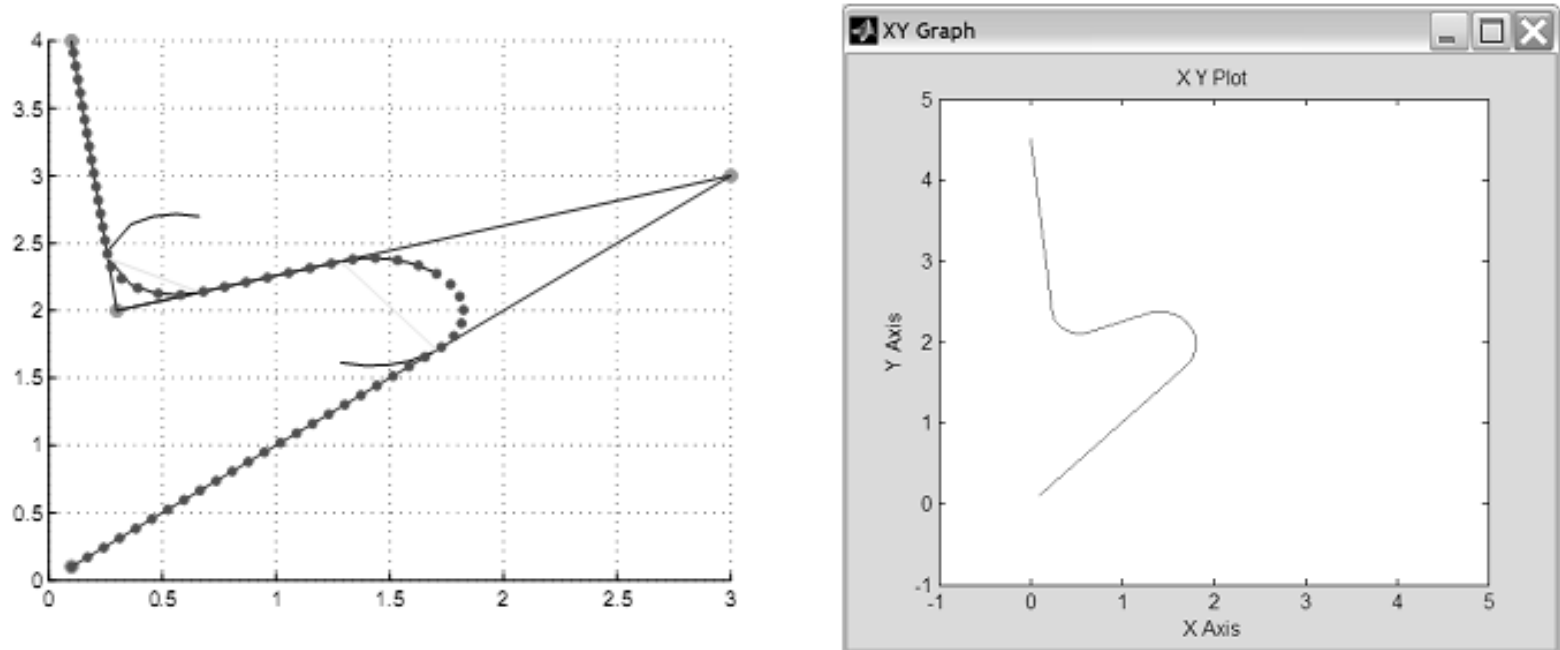

Fonte: (MELO; ROSÁRIO, 2006).

A Figura 15 apresenta os gráficos do eixos cartesianos, com seus respectivos erros. deslocamento linear de referência e dinâmico nos

Figura 15 - Gráficos do deslocamento linear de referência e dinâmico nos eixos cartesianos, com seus respectivos erros.
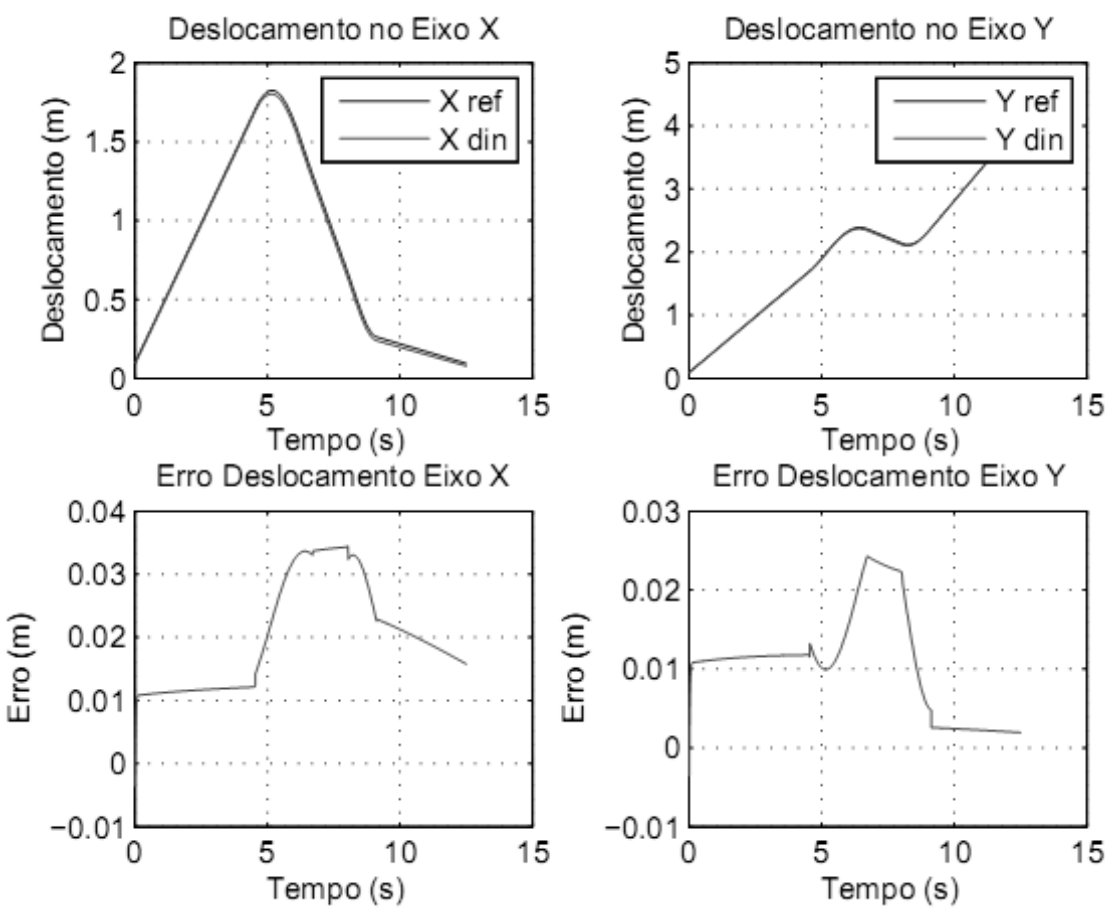

Fonte: (MELO; MANGILI JUNIOR; FLORINO, 2011). 
Outro relevante gráfico gerado pelo sistema é o gráfico da trajetória cartesiana cinemática e dinâmica do sistema robótico móvel no plano XY. A Figura 16a mostra o traçado de referência e dinâmico da trajetória do robô móvel. A Figura $16 \mathrm{~b}$ ilustra o gráfico do erro da trajetória. Notase que o erro maior na trajetória acontece no intervalo de tempo de simulação que varia de 6 a 8 segundos, corroborando os resultados obtidos anteriormente. Percebe-se também que o erro máximo da trajetória fica abaixo de $4,5 \mathrm{~cm}$, justamente num momento crítico da execução da trajetória, que é a primeira curva. Relevante notar a recuperação do sistema, apontado pelo gráfico do erro, onde o erro da trajetória tende a diminuir de intensidade. Fica claro então o excelente desempenho do sistema, resultando num nível de erro aceitável para o sistema, uma vez que a execução da trajetória foi feita com êxito.

Figura 16 - Trajetória de referência e dinâmica do robô móvel com o erro apresentado.
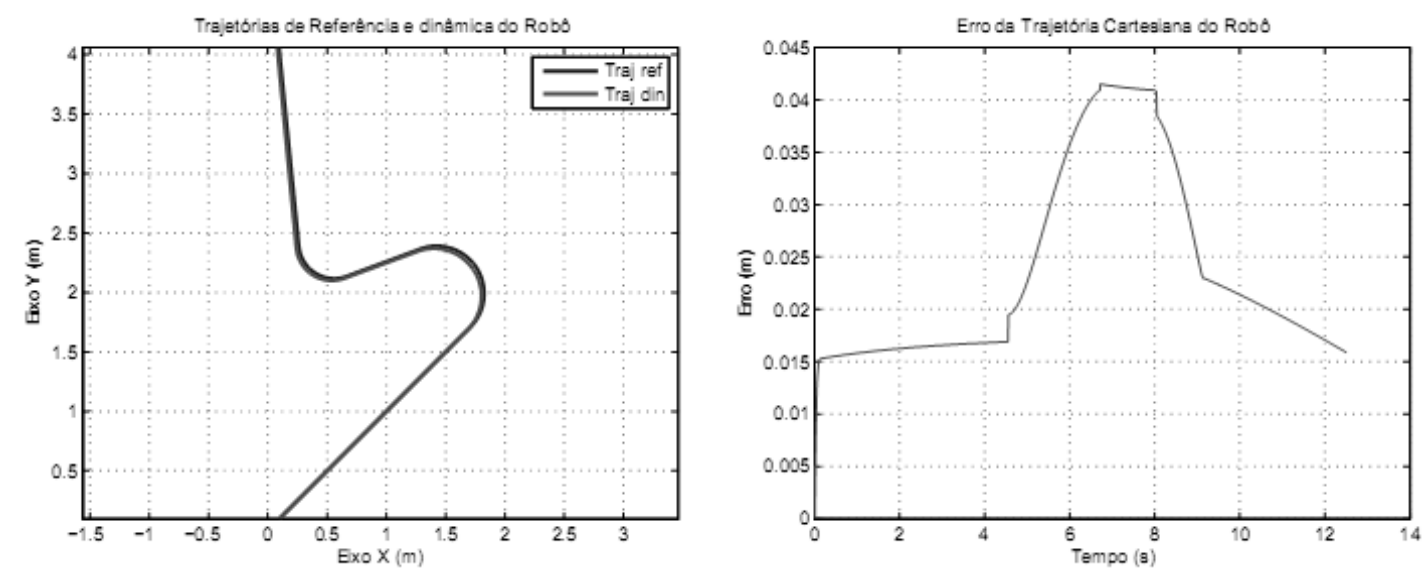

Fonte: (MELO et al., 2010).

A Figura 17 apresenta o gráfico do sinal de referência da velocidade linear da roda esquerda (motor CC esquerdo) com sua respectiva evolução dinâmica. No segundo gráfico desta mesma figura, observa-se o sinal de erro deste eixo. Pode-se notar que os erros encontrados são em forma de pulsos nos momentos exatos do começo e final de cada curva, resultante da atuação precisa do controlador no sistema dinâmico. Vale observar a estabilidade do sistema, com velocidade linear dinâmica muito próxima da sua referência. Não foi apresentada aqui a análise do eixo direito pois apresenta o mesmo perfil em termos de controle do descrito no eixo esquerdo. 
Figura 17 - Gráfico da velocidade linear na roda esquerda e do erro.
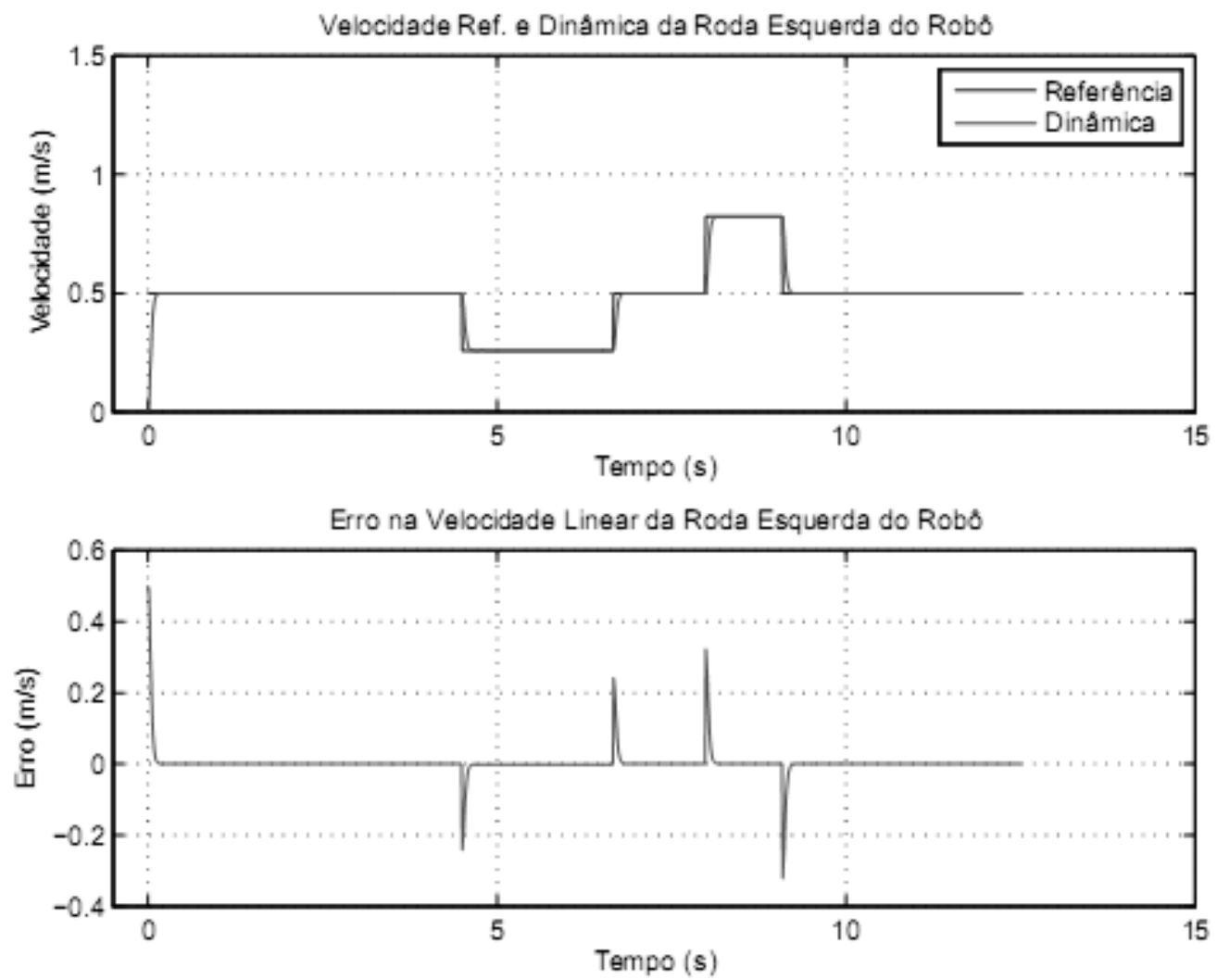

Fonte: (MELO; MANGILI JUNIOR; FLORINO, 2011).

Neste estudo de caso comprovou-se, através das análises gráficas apresentadas, a eficácia do controlador adotado para o sistema robótico móvel proposto. Comprovou-se também, a utilidade do módulo analisador gráfico na análise dos parâmetros cinemáticos e dinâmicos adotados no sistema.

Com uma velocidade linear constante aplicada ao sistema robô móvel, todos os resultados foram satisfatórios, atendendo perfeitamente as expectativas do projeto do controlador, descrito anteriormente. Assim sendo, o sistema simulador demonstrou sua aplicabilidade no sentido de prover todas as variáveis cinemáticas e dinâmicas do sistema em simulação em tempo real, deixando tudo pronto para a prototipagem rápida no sistema robótico móvel.
Prototipagem rápida nos sistemas robóticos móveis

A utilização da técnica da prototipagem rápida nos sistemas robóticos móveis foge do escopo tradicional utilizado na engenharia mecânica, como pode ser visto na seção anterior, e entra em novo campo de pesquisa e desenvolvimento para projetos de sistemas mecatrônicos de robôs móveis (MELO; MANGILI JUNIOR; FLORINO, 2011).

Neste sentido, a prototipagem rápida destes sistemas está associada não somente ao projeto do sistemafísico,masprincipalmenteàsimplementações experimentais nos campos de hardware e software do sistema robótico. A arquitetura de hardware do sistema proposto é fundamental que seja aberta e flexível no sentido de se efetuar as modificações 
necessárias para otimização do sistema, conforme apresentada anteriormente.

O sistema de controle embarcado do robô móvel, no contexto da prototipagem rápida, pode ser elaborado em simuladores e testados todos os parâmetros para ajustes que se fizerem necessários de acordo com o sistema físico a ser implementado, a arquitetura de hardware, os atuadores e os sensores.

Desta feita, no contexto deste trabalho, a prototipagem rápida é então a metodologia que permite a criação de um ambiente virtual de simulação para o projeto de um controlador para robôs móveis. Depois de testado e validado no simulador, o sistema de controle é programado na memória da placa de controle do robô móvel. Desta maneira, uma economia de tempo e material é conseguida, validando primeiramente todo o modelo virtualmente para depois se operar a implementação física do sistema.

Figura 18 - Simulação HIL do sistema robótico móvel.

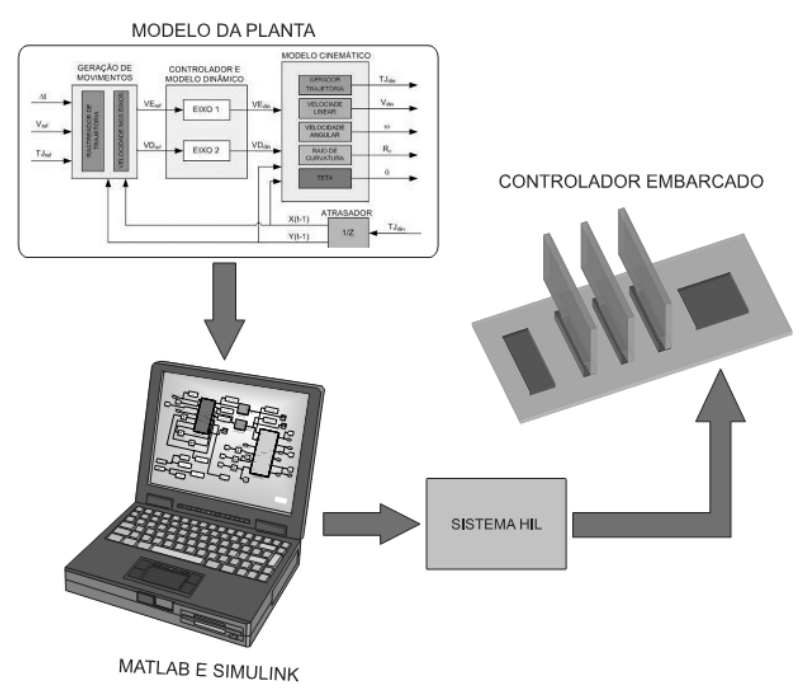

Fonte: (MELO et al., 2010).

A simulação HIL (hardware-in-the-loop) é uma forma de simulação em tempo real. Ela difere das simulações em tempo real tradicionalmente encontradas, pela adição de um componente "real" no laço de testes. Este componente pode ser uma unidade de controle eletrônico (ECU - Electronic controle unit) utilizado em sistemas embarcados automotivos ou aeroespaciais, um motor automotivo real, uma placa de controle embarcado utilizada em sistemas robóticos móveis, etc. A Figura 18 ilustra a utilização da técnica de simulação HIL para simulação em tempo real do sistema robótico móvel proposto (MELO et al., 2010).

\section{Conclusões}

Uma das principais motivações deste trabalho é propiciar um ambiente virtual que facilite o desenvolvimento de protótipos de sistemas embarcados, enfatizando a implementação de ferramentas que permitam a simulação das condições cinemáticas, dinâmicas e de controle, com monitoração em tempo real de todos pontos relevantes do sistema. Desta monta, a proposta de um simulador virtual de sistemas robóticos móveis é apresentada juntamente com técnicas de prototipagem rápida. Foi dado ênfase no projeto do simulador virtual cinemático e dinâmico de robôs móveis, onde são testadas a eficácia do projeto do controlador.

Neste contexto, a prototipagem rápida é a tecnologia que permite, em conjunto com um ambiente virtual de simulação, o desenvolvimento de um projeto de um controlador para robôs móveis. Depois de testado e validado no simulador, o sistema de controle é programado na memória da placa de controle do robô móvel. Desta feita, uma economia de tempo e material é conseguida, validando primeiramente todo o modelo virtualmente para depois se operar a implementação física do sistema.

O ambiente proposto pode também ser utilizado como uma ferramenta de apóio didático em laboratórios de graduação e pós-graduação de engenharia. $\mathrm{O}$ simulador de trajetória cinemática e dinâmica de sistemas robóticos móveis também 
é uma contribuição para o meio tecnológico de estudos robóticos. Finalmente, pode-se citar como contribuição ao meio científico, a geração de artigos publicados em revistas e eventos científicos da área, de âmbito nacional e internacional.

São aspectos promissores do ambiente proposto:

- Flexibilidade - há uma grande variedade de configurações possíveis na implementação de soluções para diversos problemas associados com desenvolvimento de protótipos de sistemas embarcados.

- Adaptação a tarefa - apenas os recursos necessários a execução de uma tarefa são utilizados. Sistemas mais simples são tratados com versões mais simples do ambiente proposto, evitando-se perda de recursos.

- Ambiente aberto - permite que seus blocos componentes sejam totalmente acessados e eventualmente modificados para agregar novas soluções

- Facilidade de expansão - novos recursos podem ser facilmente adicionados a um projeto, permitindo a adição de novas funcionalidades.

- Possibilidade de controle supervisório de vários protótipos simultaneamente.

- Custo relativamente baixo.

\section{Referências}

DUDEK, G.; JENKIN, M. Computational principles of mobile robotics. Cambridge: Press Syndicate of the University of Cambridge, 2000.

LEONARD, J. J.; DURRANT-WHYTE, H. F. Directed sonar sensing for mobile robot navigation. London: Springer, 1992.

MELO, L. F. Proposta de Simulador Virtual para Sistema de Navegação de Robôs Móveis Utilizando Conceitos de Prototipagem Rápida. Campinas: UNICAMP, 2007. 293 f. Tese (Doutorado em Engenharia Mecânica) - Programa de Pós-Graduação em Engenharia Mecânica, Faculdade de Engenharia Mecânica, Universidade Estadual de Campinas, Campinas, 2007.
MELO, L. F.; MANGILI JUNIOR, J. F. Trajectory planning for nonholonomic mobile robot using extended Kalman filter. Mathematical Problems in Engineering, Toronto, v. 2010, p. 1-22, 2010.

MELO, L. F.; MANGILI JUNIOR, J. F.; DIAS NETO, F. C.; ROSARIO, J. M. Virtual simulator for design of mobile robot control and navigation systems. In: BARRERA, A. (Org.). Mobile robots navigation. Viena: INTECH, 2010. v. 1, p. 447-467. 2010.

MELO, L. F.; MANGILI JUNIOR, J. F.; FLORINO, J. A. C. Rapid prototyping for mobile robots embedded control systems. In: HOQUE, M. E. Advanced applications of rapid prototyping technology in modern engineering. Viena: INTECH, 2011. v. 1. p. 225-242. 2011.

MELO, L. F.; ROSÁRIO, J. M. A proposal for a hybrid opened archtecture with hardware reconfigurable control applied in mobile robots. In: IEEE International Conference on Robotic and Bionemetics, 2006, Kunming. Proceedings of IEEE ROBIO 2006. Kunming: IEEE, 2006. v. 1, p. 1101-1106.

SARCINELLI FILHO, M.; SCHNEEBELI, H. A.; BASTOS FILHO, T. F.; MACHADO, É. L. Um ambiente de prototipagem rápida para o desenvolvimento de algoritmos de navegação de robôs móveis baseada em visão. Anais do XIV Congresso Brasileiro de Automática CBA 2002; 2002; v. 1; p. 1549-1554.; Natal - RN.

TEXAS Instrument. DSP. USA, 2007. Disponível em: http://www.dsp.ti.com. Acesso em: 13 mar. 2011.
Recebido em 23 Abril 2012-Received on April 23, 2012. Aceito em 25 Julho, 2012 - Accepted on July 25, 2012. 